\title{
Síntesis de pigmentos cerámicos mediante radiación microondas
}

\author{
J.CALBO, C. GARGORI, S. SORLÍ, J. BADENES, M.A. TENA, G. MONRÓS. \\ U.P. de Química Inorgánica i Orgánica, \\ Unidad de Química Inorgánica Medioambiental y Materiales Cerámicos, \\ Universitat Jaume I, Castellón (España).
}

\begin{abstract}
En el presente trabajo se ha analizado el efecto de la calcinación rápida dieléctrica con horno microondas sobre el desarrollo de pigmentos cerámicos para coloración de baldosas cerámicas. Para ello se han preparado cuatro composiciones de diferentes pigmentos cerámicos (negro de ferrocromita de cobalto, rosa de manganeso-alúmina, coral de hierro en circón y rosa de cromo estaño), empleando diferentes métodos de síntesis (ruta cerámica y métodos no convencionales), y han sido calcinadas en horno multimodo microondas, de $1250 \mathrm{~W}$ de potencia y $2450 \mathrm{MHz}$ de frecuencia, y en horno eléctrico tradicional. Los resultados obtenidos indican que la efectividad de la cocción con microondas depende del grado de homogeneidad alcanzado en los diferentes métodos de síntesis, de la microestructura de los polvos y del sistema pigmentante considerado.
\end{abstract}

Palabras clave pigmento cerámico, microondas.

Synthesis of ceramic pigments employing microwave radiation

Effect of microwave radiation on the development of ceramic pigments for tiles has been studied. Four compositions corresponding to different ceramic pigments (chrome-tin pink, cobalt-ferrite black, pink coral of iron in zircon and pink of chromium-tin sphene) have been prepared. Compositions using several synthesis methods (ceramic route and nonconventional methods) have been prepared and fired by microwave irradiation in a multimode, $1250 \mathrm{~W}$ power and 2450 $\mathrm{MHz}$ frequency, microwave equipment, and by conventional electric kiln. Results show that the efficiency of microwave firing show a great dependence on the mixing degree of precursors attained on the synthesis methods carried out, on microstructural parameters of powders and on the considered pigmenting system.

Keywords: Ceramic pigment, microwave.

\section{INTRODUCCIÓN}

Desde hace tiempo se viene aplicando la técnica calefacción rápida dieléctrica mediante horno de microondas a materiales, así como en tecnologías del medioambiente (descontaminación de suelos, vitrificación de residuos radiactivos, recuperación de petróleo) y también en la síntesis química convencional (química de materiales, de coordinación y organometálica) (1).

Se trata de una técnica de calefacción volumétrica frente a los sistemas de transmisión de calor conductivos o convectivos de tipo superficial, utilizando la posibilidad de algunos líquidos o sólidos polarizables de transformar la energía electromagnética en calor asociado a la resistencia de estos materiales a los movimientos inducidos por el campo externo (traslacionales de las cargas libres o ligadas a iones o electrones de cambios de orientación de los dipolos existentes tanto permanentes como inducidos), que provocan la aparición de fuerzas elásticas y de fricción. La calefacción microondas presenta selectividad en la calefacción lo que permite actuar específicamente sobre alguno de los componentes del sistema, además, y según algunos autores existe el llamado "efecto microondas" o efecto específico asociado a la propia radiación y no meramente térmico, descrito por Pagnota en la modificación del equilibrio de mutarrotación $\alpha$-D-glucosa $\leftrightarrow \alpha$-L-glucosa en medio EtOH: $\mathrm{H}_{2} \mathrm{O}=1: 1$, que estabiliza una mayor cantidad el isómero $\alpha-\mathrm{D}$ glucosa que la cocción tradicional no pudiéndose asociar a efectos térmicos. Sin embargo, también presenta dificultades importantes, relacionadas con la dificultad de una irradiación homogénea de la muestra, aunque los sistemas monomodo hayan relativizado este problema, y el carácter selectivo de la calefacción hace que aparezcan "puntos calientes" en el sistema, dificultando la reproducibilidad del procesado. De este modo, la homogeneidad en la muestra irradiada favorece el calentamiento de la misma, ya que los componentes "fríos" se podrán calentar mediante conducción desde los componentes "calientes", resultando esto más difícil cuando el material es heterogéneo $(1,2,3)$.

Atendiendo a parámetros de difusión térmica, un material se caracteriza por poseer una permitividad relativa compleja $\varepsilon=\varepsilon^{\prime}$-i $\varepsilon^{\prime \prime}$, siendo $\varepsilon^{\prime}$ la parte real o "constante dieléctrica" y $\varepsilon$ " la parte imaginaria o "factor de pérdida dieléctrica". Los mecanismos de disipación de energía en forma de calor en el material, vienen dados por el factor de disipación, denominado "tangente de pérdida" ( $\tan \delta)$, que se define como el cociente $\left(\varepsilon^{\prime \prime} / \varepsilon^{\prime}\right)$. 
Muchos materiales cerámicos son materiales aislantes que presentan cierta inercia al tratamiento con microondas $(2,3)$, de manera que a temperatura ambiente son transparentes a la radiación microondas con bajas tangentes de pérdida que van aumentando lentamente con la temperatura, hasta alcanzar una temperatura crítica a partir de la cual el material empieza a absorber la radiación de forma eficiente y creciente, provocando un aumento súbito de la temperatura. Así se comportan $\mathrm{SiO}_{2}$ $\mathrm{Al}_{2} \mathrm{O}_{3^{\prime}}, \mathrm{MgO}$ o los vidrios de sílice. Además la presencia de impurezas modifica seriamente su comportamiento: así la alúmina $99 \%$ de pureza que presenta una temperatura crítica de $400^{\circ} \mathrm{C}$ y velocidades de aumento bajas, $\tan \delta=0,02$ a $1200^{\circ} \mathrm{C}$, al aumentar las impurezas introducidas por mecanismos de disolución sólida en el retículo, caso de alúmina $97 \%$, el material presenta una temperatura crítica inferior a $\operatorname{los} 100^{\circ} \mathrm{C}$ y crecimiento rápido de la susceptibilidad, $\tan \delta=0,05$ a $1000^{\circ} \mathrm{C}$. Otros compuestos cerámicos como $\mathrm{Co}_{2} \mathrm{O}_{3^{\prime}}, \mathrm{MnO}_{2}, \mathrm{NiO}, \mathrm{Si}_{3} \mathrm{~N}_{4}$ presentan temperaturas críticas inferiores a la temperatura ambiente.

En la síntesis de pigmentos cerámicos por metodologías clásicas (4), la muestra se presenta heterogénea con particulados relativamente grandes $(1-5 \mu \mathrm{m})$ que al ser irradiados serán calentados de forma muy desigual, dependiendo de los factores disipativos de cada uno de los componentes, de forma que, frente a componentes muy calientes, habrá otros muy fríos que deben calentarse por la conducción de sus vecinos. Por tanto, la utilización de microondas en mezclas cerámicas clásicas no es previsible que permita grandes avances de reacción ni una adecuada reproducibilidad de la reacción cuando ésta se produce. Sin embargo, si se utilizan procesados de alta homogeneidad inicial (coprecipitados, geles, mezclas hidrotermales...), el efecto de la selectividad en irradiación queda compensada por la alta capacidad de difusión térmica en la micromezcla, si además en los materiales amorfos se retienen grupos $\mathrm{OH}^{-}$muy sensibles a la radiación microondas o se generan disoluciones sólidas que aumentan la sensibilidad térmica a microondas del material, cabe esperar una mayor eficacia térmica de la irradiación.

En el presente trabajo se pretende estudiar la eficacia de la cocción rápida dieléctrica con microondas en la síntesis de pigmentos cerámicos en función del grado de homogeneidad y microestructura de los polvos de partida de diferentes métodos de preparación de las muestras, así como del sistema pigmentante considerado, en relación con la cocción lenta térmica tradicional.

\section{PROCEDIMIENTO EXPERIMENTAL}

En el presente estudio se ha utilizado un horno microondas de tipo multimodo de $1250 \mathrm{~W}$ de potencia y $2450 \mathrm{MHz}$ de frecuencia de salida, para la calcinación de pigmentos cerámicos de diferentes familias: negro de ferrocromita de cobalto y magnesio, rosa de manganeso-alúmina, coral de hierro en circón y rosa de cromo estaño $(5,6)$.

En el caso del pigmento negro de ferrocromita de cobalto y magnesio se prepararon tres muestras mediante diferentes métodos de síntesis de la muestra precursora (cerámico, coprecipitación y síntesis hidrotermal a $40 \mathrm{~atm}$.) con el fin de estudiar la influencia de las características de los precursores en la calcinación por microondas.

Para los pigmentos de las familias rosa de manganesoalúmina, coral de hierro en circón y rosa de cromo estaño, se prepararon las muestras mediante un método no convencional de coprecipitación o de formación de poliésteres (ruta Pechini), calcinándose posteriormente en horno microondas y en horno eléctrico, con el fin de observar las diferencias existentes entre los dos tipos de calcinación.

\subsection{Negro espinela de ferrocromita de cobalto y magnesio $\mathrm{Co}_{0.5} \mathrm{Mg}_{0.5} \mathrm{Fe}_{1.9} \mathrm{Cr}_{0.1} \mathrm{O}_{4}$.}

Se preparó una única composición del pigmento $\mathrm{Co}_{0.5} \mathrm{Mg}_{0.5} \mathrm{Fe}_{1.9} \mathrm{Cr}_{0.1} \mathrm{O}_{4}$ mineralizado con un $20 \%$ molar de una mezcla $2 \mathrm{NaCl}: \mathrm{NaF}$, mediante tres métodos de síntesis: método tradicional cerámico, coprecipitación con $\mathrm{NH}_{3}$ y síntesis hidrotermal.

Estas espinelas producen coloraciones negras de alta calidad (DCMA 13-39-9 y 13-40-9) y se obtienen por calcinación a temperaturas entre 1100 y $1200^{\circ} \mathrm{C}$, con retenciones entre 2 y 6 horas a la temperatura máxima, dependiendo del sistema mineralizador utilizado. La utilización de métodos de síntesis basados en la coprecipitación o de mezclas hidrolizadas de forma controlada y conjunta de alcóxidos permite disminuir esta temperatura a 1000 y $800^{\circ} \mathrm{C}$ respectivamente. El análisis de estos materiales como pigmento cerámico por calcinación convencional en horno eléctrico se analiza en un anterior trabajo de los autores (7).

En el método cerámico $\mathrm{CE}$, una mezcla de los óxidos precursores $\left(\mathrm{Cr}_{2} \mathrm{O}_{3}, \mathrm{Fe}_{2} \mathrm{O}_{3^{\prime}} \mathrm{MgO}, \mathrm{Co}_{3} \mathrm{O}_{4}\right.$ suministrados por PANREAC) en cantidad exacta para obtener $10 \mathrm{~g}$ decomposición final $\mathrm{Co}_{0.5} \mathrm{Mg}_{0.5} \mathrm{Fe}_{1.9} \mathrm{Cr}_{0.1} \mathrm{O}_{4^{\prime}}$, fue molturada y homogeneizada en molino de bolas de tipo planetario, utilizando acetona como medio dispersante. Tras la eliminación por evaporación de la acetona residual se obtuvo el polvo seco para la posterior calcinación.

En el método de coprecipitación $\mathrm{CO}$, los precursores $\left(\mathrm{CH}_{3} \mathrm{COO}\right)_{2} \mathrm{Mg} \cdot 4 \mathrm{H}_{2} \mathrm{O}, \mathrm{Co}(\mathrm{OH})_{2^{\prime}}\left(\mathrm{CH}_{3} \mathrm{COO}\right)_{3} \mathrm{Cr}, \mathrm{FeSO}_{4} \cdot 7 \mathrm{H}_{2} \mathrm{O}$ suministrados por ALDRICH) dosificados para obtener 5 $\mathrm{g}$ de producto final se disolvieron en $200 \mathrm{~mL}$ de agua y posteriormente, manteniendo agitación continua y temperatura de $70^{\circ} \mathrm{C}$, se precipitaron mediante la adición de $\mathrm{NH}_{3}$ concentrado gota a gota hasta alcanzar un $\mathrm{pH}$ 8.5. La muestra se seco en estufa a $110^{\circ} \mathrm{C}$ para obtener el polvo para la calcinación.

Por último, se utilizó el método hidrotermal HI, que denominamos así en el sentido de técnica que con un tratamiento térmico a relativamente baja temperatura, utiliza la presión generada a dicha temperatura en un reactor herméticamente cerrado para favorecer la reacción química. En este método HI se preparó un coprecipitado del mismo modo que en el método $\mathrm{CO}$, y posteriormente se llevó a un reactor a presión en el que se mantuvo por espacio de 16 horas a una temperatura de $250^{\circ} \mathrm{C}$ alcanzando una presión estable de 40 bares. Transcurrido este tiempo se extrajo la muestra del reactor y tras el secado a $110^{\circ} \mathrm{C}$ en estufa se procedió a la calcinación.

La calcinación de las muestras se realizó en un horno microondas Superser de $1250 \mathrm{~W}$ de potencia y $2450 \mathrm{MHz}$ de frecuencia de salida, con tiempos de calcinación de 30 y 60 minutos. La caracterización mediante difracción de rayos- $X$ (DRX) se realizó tanto a los polvos calcinados como a las muestras crudas, a fin de observar el grado de evolución de fases cristalinas en la formación del pigmento. La caracterización de las muestras y análisis de las fases cristalinas presentes se realizó mediante el método de polvo orientado al azar, utilizando un difractómetro Siemens D5000. 
Con el fin de evaluar el rendimiento colorimétrico obtenido, los pigmentos se esmaltaron sobre un soporte cerámico convencional (5\% de pigmento), utilizando un vidriado cristalino transparente de monococción porosa convencional de composición base $\mathrm{SiO}_{2}-\mathrm{ZnO}-\mathrm{CaO}$ suministrado por Torrecid S.A que madura a $1080^{\circ} \mathrm{C}$. La caracterización óptica de las plaquetas esmaltadas y los parámetros colorimétricos siguiendo la convención CIE-L*a* $\mathrm{b}^{*}$ fueron obtenidas con un espectrofotómetro UV-V-NIR Perkin-Elmer Lambda 2000 utilizando la técnica de reflectancia difusa, en este sistema $L^{*}$ mide la claridad, $a^{*}$ la proporción de color verde(-) $\rightarrow$ rojo $(+)$, $\mathrm{y} \mathrm{b}^{*}$ la de azul(-) $\rightarrow$ amarillo(+) (8). Por último, para estudiar la microestructura de las muestras obtenidas, se obtuvieron micrografías de las muestras recubiertas por sputtering de grafito sobre porta de aluminio, en un microscopio electrónico LEO.2i equipado con un sistema de microanálisis Oxford.

\subsection{Coral de hierro en circón $0,25 \mathrm{Fe}_{2} \mathrm{O}_{3}-\mathrm{ZrSiO}_{4}$.}

El pigmento rojo de hierro en circón (DCMA 14-44-5) se prepara en las mezclas cerámicas entre 900 y $1000^{\circ} \mathrm{C}$, con retenciones de entre 2 y 4 horas a la temperatura máxima y añadiendofluorurosinorgánicos comoagentesmineralizadores. El pigmento basado en la el encapsulamiento de nanocristales de hematita en los cristales de circón se puede obtener sin mineralización y con mejores eficiencias de encapsulamiento utilizando métodos basados en la coprecipitación (9). Se preparó una única composición $0,25 \mathrm{Fe}_{2} \mathrm{O}_{3}-\mathrm{ZrSiO}_{4}$ mediante el método de coprecipitación CO. Los precursores (Sulfato de hierro(II) heptahidrato, oxicloruro de circonio octahidrato, suministrados por ALDRICH y sílice coloidal de DEGUSSA) en la cantidad exacta para preparar $5 \mathrm{~g}$ de composición final $0,25 \mathrm{Fe}_{2} \mathrm{O}_{3}-\mathrm{ZrSiO}_{4^{\prime}}$ se disolvieron en $200 \mathrm{~mL}$ de agua a una temperatura de $70^{\circ} \mathrm{C}$ manteniendo agitación continua y posteriormente se precipitaron con $\mathrm{NH}_{3}$ concentrado añadido gota a gota sobre la disolución en agitación continua a $70^{\circ} \mathrm{C}$. El precipitado obtenido fue lavado con mezcla $\mathrm{NH}_{3}(33 \%)$ : Agua en proporción 1:5 y los mineralizadores $\mathrm{NaF}: 2 \mathrm{NaCl}$, se añadieron en una proporción del $20 \%$ molar al precipitado emulsionado en agua. Una vez preparada, la muestra se secó en estufa a $110^{\circ} \mathrm{C}$.

Los polvos obtenidos se homogeneizaron en mortero de ágata, posteriormente se separó una parte de la muestra para calcinarla según el método cerámico en horno eléctrico durante 2,5 horas a $800^{\circ} \mathrm{C}$. La muestra restante se separó para realizar dos calcinaciones en horno microondas, durante $30 \mathrm{y}$ 60 minutos.

Los polvos obtenidos fueron estudiados por difracción de rayos $\mathrm{X}$ mediante el método de polvo orientado al azar, con la finalidad de estudiar las diferentes fases cristalinas formadas durante la calcinación. Asimismo, las muestras se esmaltaron en le vidriado indicado en el apartado 3.1 para los estudios colorimétricos.

\subsection{Rosa de Manganeso-Alúmina $\mathrm{Mn}_{0.3} \mathrm{Al}_{0.7} \mathrm{O}_{3}$.}

El rosa de manganeso alúmina (DCMA 3-04-5) es una disolución sólida de $\mathrm{Mn}^{3+}$ en red de corindón que se obtiene por procedimientos cerámicos de reacción en estado sólido entre 900 y $1000^{\circ} \mathrm{C}$ con retenciones entre 3 y 6 horas (10). Se preparó una única composición $\mathrm{Mn}_{03} \mathrm{Al}_{07} \mathrm{O}_{3}$ mediante el método del poliéster o Pechini. Los precursores (Carbonato de Manganeso (II) y acetato básico de Aluminio de ALDRICH) en la cantidad exacta para preparar $5 \mathrm{~g}$ de composición final $\mathrm{Mn}_{0.3} \mathrm{Al}_{0.7} \mathrm{O}_{3^{\prime}}$, se disolvieron en $200 \mathrm{~mL}$ de una disolución de ácido cítrico $1 \mathrm{M}$ a $70^{\circ} \mathrm{C}$ y en agitación continua, posteriormente se añadieron 0,3 moles de etilenglicol como agente de poliesterificación y se mantuvo la agitación y la temperatura de $70^{\circ} \mathrm{C}$ durante una hora.

Una vez preparada la muestra, se secó en estufa a $110^{\circ} \mathrm{C}$ hasta sequedad total del poliéster, posteriormente se realizó el tratamiento de "quemado" del poliéster obtenido a $350^{\circ} \mathrm{C}$ durante 1hora.

Los polvos obtenidos se molturaron en mortero de ágata con el fin de homogeneizarlos, posteriormente se separó una parte de la muestra para calcinarla en horno eléctrico 2,5 horas a $800^{\circ} \mathrm{C}$. La muestra restante fue irradiada en horno microondas. Los polvos resultantes se analizaron mediante las técnicas ya indicadas en el apartado 3.1.

\subsection{Rosa de Esfena de Cromo-Estaño.}

El rosa de cromo estaño (DCMA 12-25-5) es una disolución sólida de cromo(IV)enla red esfena de cromo-estaño $\left(\mathrm{CaSnSiO}_{5}\right)$, donde substituye al estaño en coordinación octaédrica (11). La esfena se obtiene por vía cerámica por calcinación de los óxidos a temperaturas entre $1200-1400^{\circ} \mathrm{C}$ con retenciones entre 3 y 6 horas dependiendo de los precursores y la mineralización utilizada. Mediante métodos químicos de mezclado como la coprecipitación se puede obtener a $1100^{\circ} \mathrm{C}$ con retenciones de 6 horas $(10,11)$. Se preparó una única composición mediante el método de coprecipitación. Los precursores (Cloruro de estaño(II), cloruro de calcio, sílice coloidal y cromato plomo(II) suministrados por ALDRICH) en las cantidades exactas para producir $5 \mathrm{~g}$ de la composición final $\mathrm{CaSnSiO}_{5}$, se disolvieron en $200 \mathrm{~mL}$ de agua y se precipitaron manteniendo agitación continua a $70^{\circ} \mathrm{C}$ añadiendo amoniaco concentrado gota a gota hasta $\mathrm{pH} 8,5$. La muestra fue secada en estufa a $110^{\circ} \mathrm{C}$.

Los polvos obtenidos se molturaron en mortero de ágata con el fin de homogeneizarlos, se separó una parte de la muestra para calcinarla en horno eléctrico 2,5 horas a $800^{\circ} \mathrm{C}$. La muestra restante se trató en horno microondas. Los polvos resultantes se analizaron mediante las técnicas ya indicadas en el apartado 3.1.

\section{RESULTADOS Y DISCUSIÓN}

3.1. Influencia del método de síntesis en la eficacia de la calcinación por microondas en el negro de ferrocromita de cobalto y magnesio $\mathrm{Co}_{0.5} \mathrm{Mg}_{0.5} \mathrm{Fe}_{1.9} \mathrm{Cr}_{0.1} \mathrm{O}_{4}$.

De acuerdo con la descripción experimental del apartado 3.1., el pigmento negro espinela $\mathrm{Co}_{0.5} \mathrm{Mg}_{0.5} \mathrm{Fe}_{1.9} \mathrm{Cr}_{0.1} \mathrm{O}_{4}$ mineralizado con un $20 \%$ molar de una mezcla $2 \mathrm{NaCl}$ : $\mathrm{NaF}$, se preparó mediante tres métodos distintos, de modo que los precursores se integraron en una mezcla física de los óxidos metálicos en el caso del método cerámico $\mathrm{CE}$, un coprecipitado de los precursores llevado a sequedad en método de coprecipitación $\mathrm{CO}$, y un coprecipitado sometido a elevadas presiones en el método hidrotermal HI.

Atendiendo a la caracterización realizada por DRX (Tabla II y Figura 3), puede observarse como la reactividad de las muestras varía sustancialmente en función del método empleado para la preparación de los precursores. En las muestras crudas se observa la presencia de los óxidos 
precursores en la muestra cerámica cruda (hematita, periclasa y óxido de cobalto), en cambio, en las muestras coprecipitadas e hidrotermal se observa la cristalización de cloruro amónico en el secado del precipitado amoniacal, junto a picos de la fase maghemita. En la Figura 1.a se observa que la muestra procesada por el método CE no presenta evolución en el difractograma realizado tras la calcinación con microondas: los óxidos de hierro, cobalto, cromo y magnesio realtivamente fríos a la radiación microondas no han alcanzado la temperatura suficiente para desarrollar la reacción en estado sólido. Sin embargo, en las muestras $\mathrm{CO}$ y $\mathrm{HI}$ se puede observar la desaparición del cloruro amónico, descompuesto en la calcinación, y la cristalización de ferrocromita (picos F en la Tabla I y Figura 1), al calcinar mediante horno microondas.

Esta diferencia de reactividad en las muestras puede explicarse mediante el efecto de micromezcla: las muestras

\section{n' cuentas}

(u.a)

(a) $\mathrm{CE}$
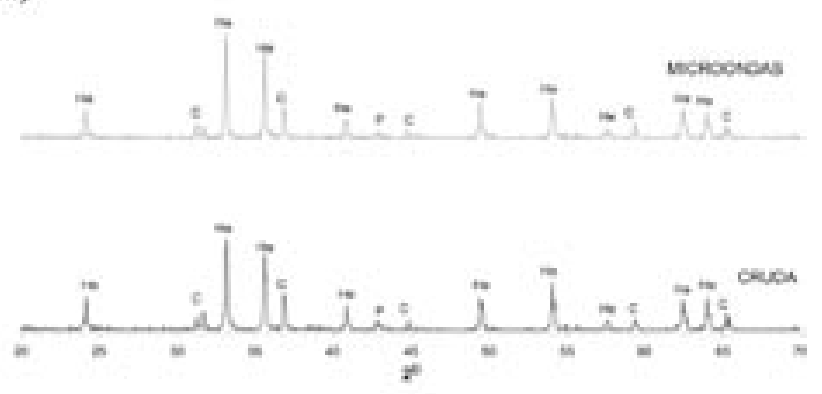

" cuentas

(b) co

(u,a)

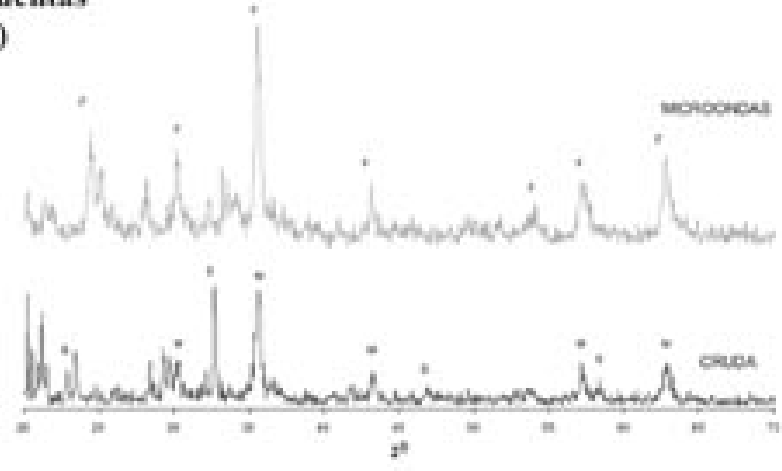

(c) HI 48 atm.

\section{$n^{\circ}$ cuentas}

(u.a)

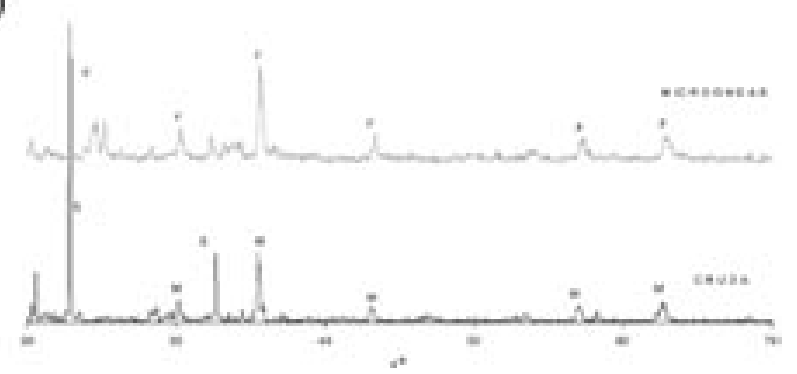

Fig. 1- Evolución de los difractogramas de rayos $\mathrm{x}$ de las muestras. FASES CRISTALINAS: C(Óxido de cobalto $\left.\mathrm{Co}_{3} \mathrm{O}_{4}\right), \mathrm{He}(\mathrm{Hematita} \alpha$ $\left.\mathrm{Fe}_{2} \mathrm{O}_{3}\right), \mathrm{P}($ Periclasa $\mathrm{MgO}), \mathrm{F}\left(\right.$ Magnesioferrita $\left.\mathrm{MgFe}_{2} \mathrm{O}_{4}\right), \mathrm{M}($ Maghemita $\left.\mathrm{Fe}_{2} \mathrm{O}_{3}\right), \mathrm{S}\left(\right.$ Cloruro amónico $\mathrm{NH}_{4} \mathrm{Cl}$ ).
TABLA I. CONSTANTE DIELÉCTRICA (E) Y FACTOR DE DISIPACIÓN (TAN $\Delta$ ) MEDIDOS A $20^{\circ} \mathrm{C}$.

\begin{tabular}{|c|c|c|}
\hline COMPUESTO & $\varepsilon^{\prime}$ & $\tan \delta \times 10^{4}$ \\
\hline $\mathrm{H}_{2} \mathrm{O}$ & 76,7 & 1570 \\
\hline Vidrio $\mathrm{SiO}_{2}$ & 3,8 & $2-8$ \\
\hline $\mathrm{Al}_{2} \mathrm{O}_{3}$ & $5-8$ & 4 \\
\hline $\begin{array}{c}\mathrm{Mg}_{2} \mathrm{SiO}_{4} \\
\text { (forsterita) }\end{array}$ & 6,2 & $2-40$ \\
\hline $\begin{array}{c}\mathrm{MgSiO}_{3} \\
(\text { enstatita })\end{array}$ & $5,5-7,5$ & $2-80$ \\
\hline $\begin{array}{c}\mathrm{ZrSiO} \\
\text { (circón) }\end{array}$ & $7,1-10,5$ & $3-200$ \\
\hline Porcelana & $6-8$ & \\
\hline
\end{tabular}

con un menor tamaño de partícula y mayor homogeneidad (con posibilidad formación de disoluciones sólidas en el material hidroxilado amorfo que aumentan la sensibilidad a la radiación microondas), como es el caso de las muestras preparadas por coprecipitación $\mathrm{CO}$ y por el procesado hidrotermal HI, son más dsensibles a la radiación microondas y se calientan de forma más eficiente, permitiendo así una mayor reactividad del sistema. En efecto, en la Figura 2 se presentan las micrografías de los materiales irradiados, se observa que frente a la presencia de partículas de 3-5 $\mu \mathrm{m}$ en la muestra cerámica, en las muestras $\mathrm{CO}$ y $\mathrm{HI}$ aparecen particulados mucho más finos, entre 200-500 nm de tamaño medio, parcialmente sinterizados en el caso de la muestra $\mathrm{CO}$ y desagregados en la muestra hidrotermal.

El comportamiento colorimétrico de los materiales irradiados al ser esmaltados al $5 \%$ en el vidriado de monoporosa (Tabla I), es de color marrón en el caso de la muestra cerámica típica de la capacidad colorante de la maghemita presente en la muestra. Los materiales $\mathrm{CO}$ y $\mathrm{HI}$ presentan coloración gris oscuro, más negro en el caso de la muestra CO (menor claridad $L^{*}$ y valores mínimos de $a^{*} y$ $\left.\mathrm{b}^{*}\right)$. El mejor rendimiento colorimétrico de la muestra CO se puede asociar a su mayor tamaño de partícula, que le permite una mayor resistencia al ataque del vidriado. En el caso de las pequeñas partículas del material $\mathrm{HI}$, son disueltas en mayor proporción por el vidriado.

3.2. Comparación entre la calcinación tradicional y la calcinación por irradiación con microondas en pigmentos rosa preparados por vía homogénea.

Una de las tonalidades más complicadas de resolver en la producción de pigmentos cerámicos es la rosa-rojo. Se ha estudiado el efecto comparado de la calcinación tradicional en horno eléctrico con la irradiación con microondas para

TABLA II. EVOLUCIÓN DE LAS FASES CRISTALINAS CON LA CALCINACIÓN EN HORNO MICROONDAS Y PARÁMETROS COLORIMÉTRICOS EN LAS MUESTRAS PREPARADAS CON DISTINTOS TIPOS DE PRECURSORES

\begin{tabular}{|c|c|c|c|}
\hline MUESTRAS & $\mathrm{CE}$ & $\mathrm{CO}$ & $\mathrm{HI}$ \\
\hline SIN TRATAR & $\mathrm{C}(\mathrm{f}), \mathrm{He}(\mathrm{f}), \mathrm{P}(\mathrm{f})$ & $\mathrm{M}(\mathrm{m}), \mathrm{S}(\mathrm{m})$ & $\mathrm{M}(\mathrm{m}), \mathrm{S}(\mathrm{mf})$ \\
\hline MICROONDAS & $\mathrm{C}(\mathrm{f}), \mathrm{He}(\mathrm{f}), \mathrm{P}(\mathrm{f})$ & $\mathrm{F}(\mathrm{f}), \mathrm{S}(\mathrm{md})$ & $\mathrm{F}(\mathrm{f}), \mathrm{S}(\mathrm{md})$ \\
$\begin{array}{c}\left(\mathrm{L}^{*} / \mathrm{a}^{*} / \mathrm{b}^{*}\right) \\
\text { esmaltadas }\end{array}$ & $(46,2 / 4,4 / 9,4)$ & $(36,9 / 3,1 / 5,1)$ & $(38,9 / 3,8 / 8,0)$ \\
\hline
\end{tabular}

FASES CRISTALINAS: $\mathrm{C}\left(\right.$ Óxido de cobalto $\left.\mathrm{Co}_{3} \mathrm{O}_{4}\right), \mathrm{He}\left(\right.$ Hematita $\left.\alpha-\mathrm{Fe}_{2} \mathrm{O}_{3}\right), \mathrm{P}($ Periclasa $\mathrm{MgO}$ ), $\mathrm{F}$ (Magnesioferrita $\left.\mathrm{MgFe}_{2} \mathrm{O}_{4}\right), \mathrm{M}\left(\right.$ Maghemita $\mathrm{Fe}_{2} \mathrm{O}_{3}$ ), S(Cloruro amónico $\mathrm{NH}_{4} \mathrm{Cl}$ ), $\mathrm{NI}$ (Pico no identificado). INTENSIDAD PICOS: $\mathrm{mf}($ muy fuerte), f(fuerte), $\mathrm{m}$ (media), d(débil), md(muy débil). 
tres pigmentos rosa: (a) el pink coral, un encapsulado de hematita en matrices de circón, (b) rosa de manganesoalúmina, una disolución sólida de $\mathrm{Mn}^{3+}$ en corindón, y (c) rosa

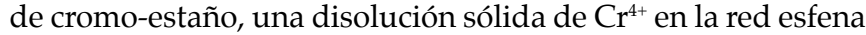
$\mathrm{CaSiSnO}_{5}$.
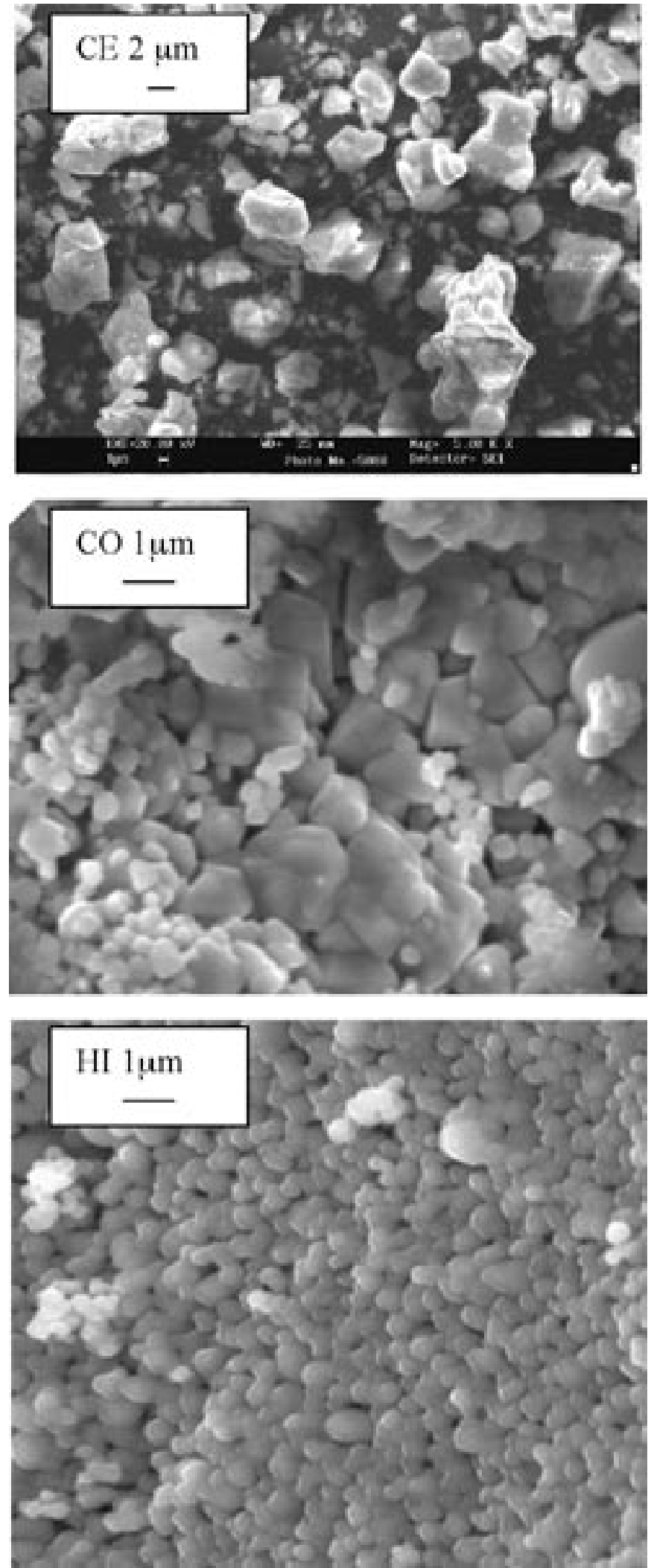

Fig. 2- Micrografías de las muestras negro espinela $\mathrm{Co}_{0.5} \mathrm{Mg}_{0.5} \mathrm{Fe}_{1.9} \mathrm{Cr}_{0.1} \mathrm{O}_{4}$.
TABLA III. CORAL DE HIERRO EN CIRCÓN. RESULTADOS DE PARÁMETROS COLORIMÉTRICOS.

\begin{tabular}{|c|c|c|}
\hline CALCINACIÓN & $\begin{array}{c}\text { Horno eléctrico. } \\
800^{\circ} \mathrm{C} / 2.5 \mathrm{~h}\end{array}$ & $\begin{array}{c}\text { Microondas } \\
60 \text { minutos }\end{array}$ \\
\hline $\mathrm{L}^{*}$ & 88,8 & 73,6 \\
\hline $\mathrm{a}^{*}$ & 1,0 & 5,3 \\
\hline $\mathrm{b}^{*}$ & 15,6 & 11,9 \\
\hline
\end{tabular}

En el caso del pigmento coral de hierro en circón, la caracterización por DRX se presenta en la Figura 3. La muestra coprecipitada presenta un difractograma amorfo (no mostrado en Figura 3). En la muestra irradiada en microondas durante 30 minutos se presenta únicamente badeleyita $M$ en equilibrio con la circona tetragonal metaestable T. En la muestra calcinada irradiada durante 60 minutos se observa la cristalización de circón $\left(\mathrm{ZrSiO}_{4}\right)$ con picos intensos, además de picos asociados a badeyelita $\left(\mathrm{ZrO}_{2}\right)$ y hematita de intensidad muy débil. La muestra calcinada en horno eléctrico presenta circona monoclínica (badeleyita $\mathrm{ZrO}_{2}$ ), cristobalita $\left(\mathrm{SiO}_{2}\right)$ y hematita pero no se detecta fase circón. Como era de esperar la muestra calcinada en horno eléctrico no reaccionada, no produce coloración al ser esmaltada al $5 \%$ en el vidriado de monoporosa, en cambio la muestra irradiada 60 minutos presenta una coloración rosa. La mejor coloración de la muestra esmaltada también se hace evidente en el espectro UV-VISNIR por reflectancia difusa de las muestras comparadas en la Figura 3, donde la muestra irradiada presenta una banda ancha asociada a $\mathrm{Fe}^{3+}$ en entorno octaédrico, centrada en trono a $500 \mathrm{~nm}$, más pronunciada. La reactividad del sistema se asocia con la presencia de circona monoclínica en el sistema, este óxido presenta una de las conductividades térmicas más bajas de todos los materiales conocidos, sólo $2 \mathrm{~W} / \mathrm{mK}$, y es
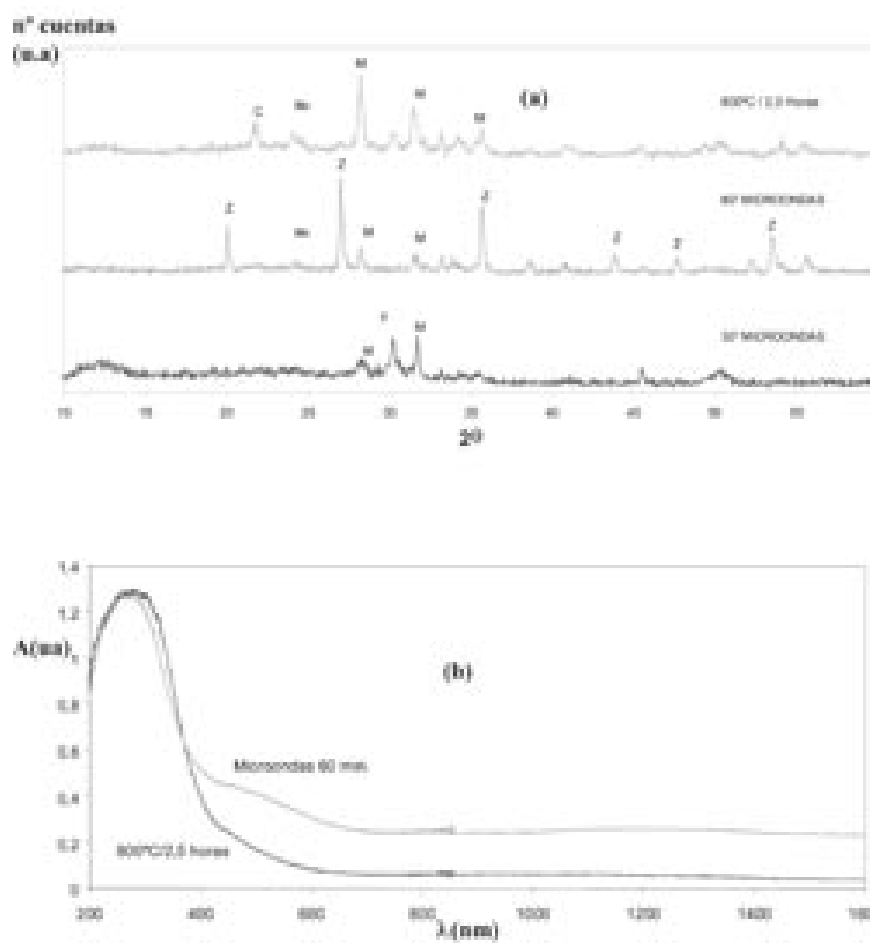

Fig. 3- Pink Koral: (a) DRX, (b) espectro UV-VIS-NIR. 

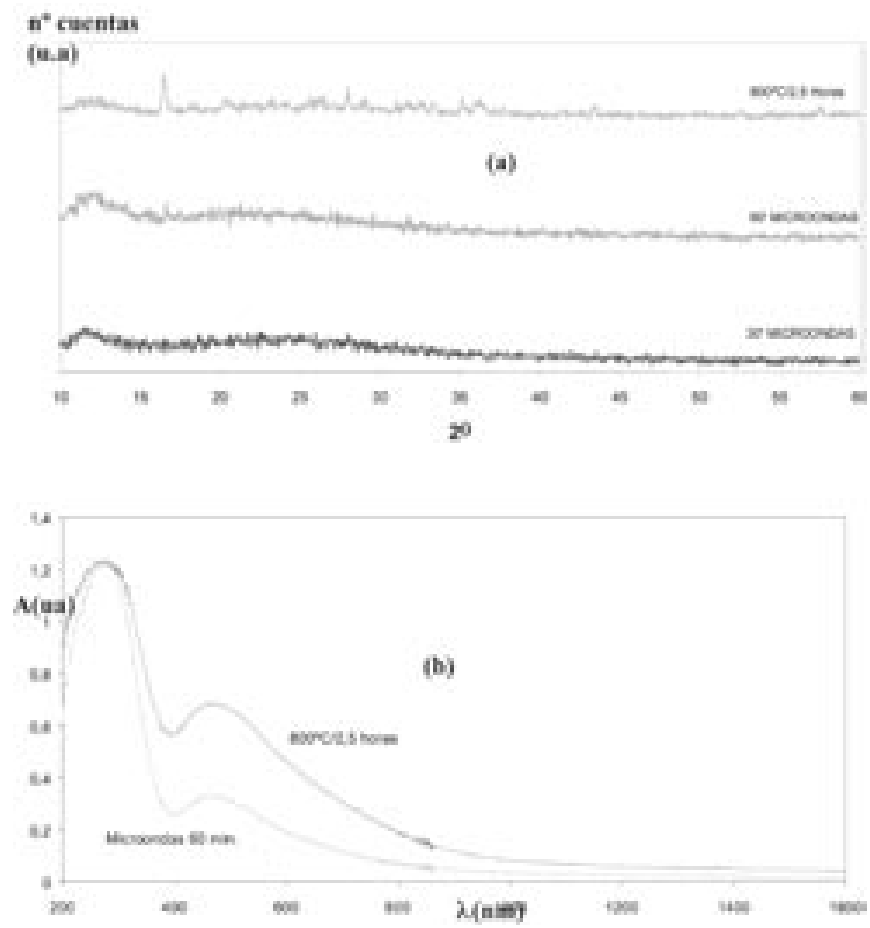

Fig. 4- Rosa de manganeso-alúmina: (a) DRX, (b) espectro UV-VISNIR.

un pobre receptor de microondas a temperatura ambiente, aunque con temperaturas críticas entre 250 y $400^{\circ} \mathrm{C}$, inferiores a la sílice. La calefacción tradicional está dificultada por la estabilización en los coprecipitados de circona tetragonal observada en la irradiación corta de 30 minutos en la Figura 3 $(12,13)$, lo mismo ocurriría en la irradiación por microondas, sin embargo, esta estabilización de la especie tetragonal en los polvos coprecipitados evita la generación de puntos calientes en la primera fase de la irradiación y al cristalizar la monoclínica, la temperatura ya supera el valor crítico por lo que la irradiación se hace efectiva.

En el caso de la muestra preparada por el método de poliéster de rosa de manganeso-alúmina, la caracterización

TABLA IV. ROSA MN-AL. EVOLUCIÓN DE LAS FASES CRISTALINAS CON LA CALCINACIÓN Y PARÁMETROS COLORIMÉTRICOS.

\begin{tabular}{|c|c|c|}
\hline CALCINACIÓN & $\begin{array}{c}\text { Horno eléctrico. } \\
800^{\circ} \mathrm{C} / 2.5 \mathrm{~h}\end{array}$ & $\begin{array}{c}\text { Microondas } \\
60 \text { minutos }\end{array}$ \\
\hline $\mathrm{L}^{*}$ & 58.9 & 78.5 \\
\hline $\mathrm{a}^{*}$ & 10.9 & 7.3 \\
\hline $\mathrm{b}^{*}$ & 10.8 & 8.6 \\
\hline
\end{tabular}

realizada por DRX se presenta en la Figura 4 . No se observa cristalización significativa en ninguna de las tres calcinaciones, presentándose un material amorfo. Únicamente en la calcinación mediante horno eléctrico se observan picos asociables a corindón. El esmaltado de las muestras produce un rendimiento colorimétrico aceptable pero más rosa (valor de $\mathrm{a}^{*}$ ) en el caso del tratamiento en horno eléctrico. La mejor coloración de la muestra esmaltada también se hace evidente en el espectro UV-VIS-NIR por reflectancia difusa de las muestras comparadas en la Figura 4, donde la muestra calcinada en horno eléctrico presenta una banda asociada a
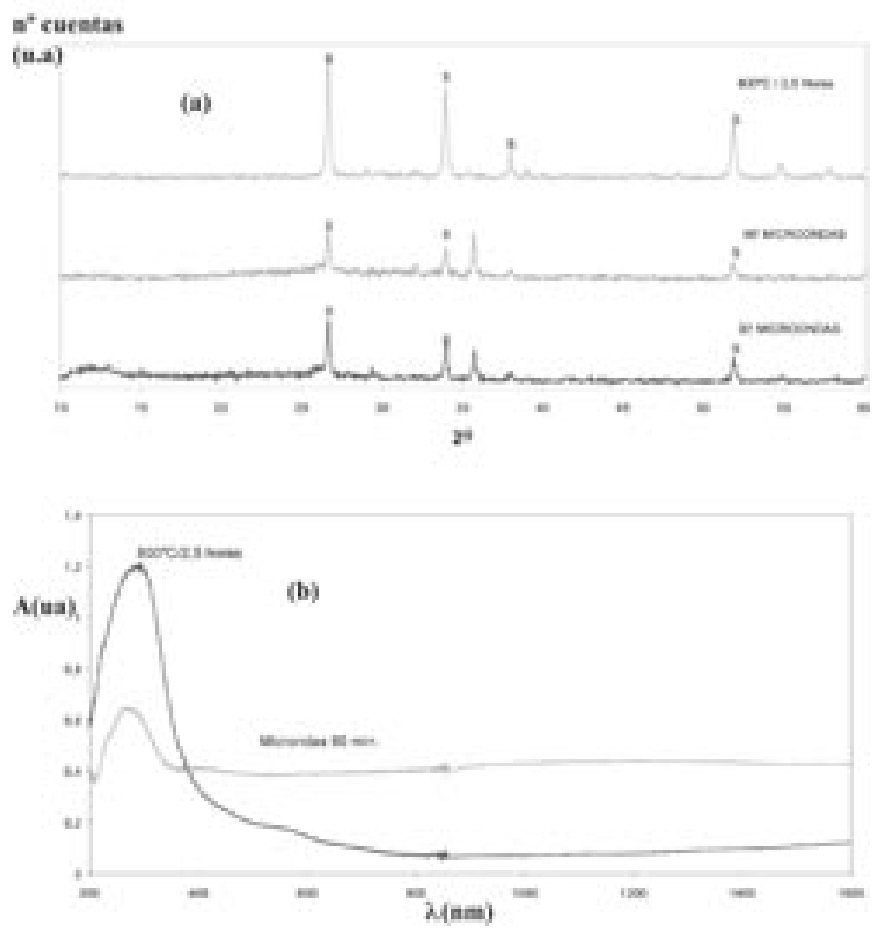

Fig. 5- Rosa de cromo-estaño: (a) DRX, (b) espectro UV-VIS-NIR.

TABla V. ROSA CROMO-ESTAÑo. RESUltados DE PARÁmEtros COLORIMÉTRICOS.

\begin{tabular}{|c|c|c|}
\hline CALCINACIÓN & $\begin{array}{c}\text { Horno eléctrico. } \\
800^{\circ} \mathrm{C} / 2.5 \mathrm{~h}\end{array}$ & $\begin{array}{c}\text { Microondas } \\
60 \text { minutos }\end{array}$ \\
\hline $\mathrm{L}^{*}$ & 84.8 & 70.4 \\
\hline $\mathrm{a}^{*}$ & 1.3 & $-1,0$ \\
\hline $\mathrm{b}^{*}$ & 9.3 & 1.6 \\
\hline
\end{tabular}

$\mathrm{Mn}^{3+}$ en entorno octaédrico, centrada en torno a $450 \mathrm{~nm}$, más pronunciada. La transparencia a la radiación microondas a bajas temperaturas del óxido de aluminio, la matriz amorfa o cristalina del material, explica el mejor comportamiento de la calcinación tradicional en este caso.

La caracterización por DRX del coprecipitado amorfo preparado en el sistema rosa de cromo-estaño se presenta en la Figura 5. En todas las muestras se detecta la cristalización de casiterita, con picos de mayor intensidad en el caso de la muestra calcinada en horno eléctrico, no se detectan picos asociables a la esfena. En ningún caso se obtiene coloración rosa aunque la coloración gris es más oscura en el caso de la muestra irradiada (Tabla IV). El espectro UV-VIS-NIR por reflectancia difusa de las muestras comparadas en la Figura 4 no presenta bandas de absorción, presentando la muestra irradiada una mayor absorbancia general asociada a la coloración oscura desarrollada. El pigmento se obtiene por el método tradicional a temperaturas mas altas $\left(1100^{\circ} \mathrm{C} / 6 \mathrm{~h}\right.$ mediante coprecipitados mineralizados), por lo que los tratamientos utilizados son insuficientes, siendo el tratamiento por irradiación menos efectivo en este caso dado el carácter frío del $\mathrm{SnO}_{2}$. 


\section{CONCLUSIONES}

Los resultados obtenidos indican que la irradiación con microondas aplicada a la obtención de pigmentos cerámicos puede ser efectiva siempre que la mezcla de precursores sea suficientementehomogénea, con el fin de conseguir un aumento de la sensibilidad a la radiación microondas por retención de grupos sensibles $\mathrm{OH}^{\text {- }}$, formación de disoluciones sólidas y calentamiento de los posibles centros poco susceptibles al calentamiento, mediante mecanismos de difusión térmica, desde los centros susceptibles. Así se comprueba en el caso de la síntesis del negro de espinela de ferrocromita de cobalto dopado con magnesio en el que la muestra preparada por vía cerámica no reacciona, en cambio, las muestras preparadas por vías homogéneas de coprecipitación o síntesis hidrotermal permiten la reactividad del sistema cristalizando la espinela.

Por otro lado, partiendo se sistemas homogéneos como coprecipitados o poliésteres, la irradiación con microondas puede ser más efectiva cuando los materiales precursores son suficientemente susceptibles a la radiación (caso del pink koral de hematita-circón), sin embargo, si el material matriz es poco susceptible a la radiación (caso de la alúmina en el rosa de manganeso-alúmina o del óxido de estaño en el rosa de esfena de cromo-estaño) la calcinación por el método tradicional es más efectiva.

\section{AGRADECIMIENTOS}

Los autores agradecen la financiación al Ministerio de Educación y Ciencia (Proyecto MAT2005-00507) y a la Fundación Caja de Ahorros de Castellón (Proyecto P1-1B200506) y la colaboración de Torrecid S.A.

\section{BIBLIOGRAFÍA}

1. (a) D.E. Clark, W.H. Sutton, Microwaves and materials, Re. Mater. Sci., 26 299-302 (1996), (b) W.R. Tinga, W.A.G. Voss, «Microwave Power Engineering, Academic Press», New York, 1968.

2. A. Díaz et al., «Técnicas no convencionales en química», Anales de la RSEQ, Abril-Julio, 1999.

3. (a) J. Portolés, «Utilización de las microondas en el procesamiento de materiales cerámicos», Cerámica Información, 189 2-25 (1993), (b) F. Bondioli, A.M. Ferrari, C. Leonelli, C. Siligardi, N.A. Hart, N.G. Evans, «The application of microwaves in the synthesis of $\mathrm{Ce} 0,9 \operatorname{Pr} 0,1^{\circ} 2$ nanostructured powders», J. Mater. Chem. 11 2620-2624 (2001).

4. (a) H. Camacho, A. García, L. Fuentes, «Study of factor affecting colour in glaze ceramics», Bol. Soc. Esp. Ceram. V., 44(1) 13-19 (2005), (b) G. Monrós, J. A. Badenes, A. García, M.A. Tena, «El color de la Cerámica», Ed. Universitat Jaume I, 2003.

5. G. Monrós, «Recenti svilupi nel settore della ricerca sui pigmenti ceramici», Ceramurgia, 5-6 175-181(2002).

6. DCMA Classification and Chemical Description of de Mixed Metal Oxide Inorganic Coloured Pigments, 2n ed. Metal oxides and ceramic colours subcommitee, Dry Color Manufacturer's Assn., Washington D.C., 1982.

7. J. Calbo, A. Mestre, A. García, M.A. Tena, M. Llusar, A. García, G. Monrós., "Multicomponent black coloured spinels from alkoxides». J. Sol-Gel Tech., 26 813-816 (2002).

8. CIE Comission International de l'Eclairage, recommendations on Uniform Color Spaces, Color Difference equations, Psychometrics Color Terms. Suplement $\mathrm{n}^{\circ} 2$ of CIE Pub. No 15 (E1-1.31) 1971, Bureau Central de la CIE, Paris (1978).

9. A. García, M. Llusar, J. Badenes, M.A. Tena, G. Monrós, «Encapsulation of hematite in zircon by microemulsion and Sol-Gel methods», J. Sol-Gel Tech, 27(3) 267-276 (2003).

10. G. Monrós, «Recent advances in the research for new ceramic pigments, Advances in Science and Technology». Science for New Technology of Silicate Ceramics, 34 255-266 (2003).

11. G. Monrós, H. Pinto, J. Badenes, M. Llusar, M.A. Tena, «Chromium(IV) stabilisation in new ceramic matrices by coprecipitation method: application as ceramic pigments», Z. Anorg. Allg. Chem, 631 2131-2135 (2005).

12. A. García, S.Sorlí, J. Calbo, M.A. Tena, G. Monrós, «Effect of the surfactant and precipitant on the synthesis of pink coral by microemulsion», J. Eur. Ceram. Soc., 23 1829-1838 (2003).

13. P. Tartaj, L.C. De Jonghe, «Preparation of nanospherical amorphous zircon powders by a microemulsion-mediated process», J. Mater. Chem 10 27862790 (2000). 